\title{
Wieso werde ich ständig übergriffig?
}

Protokoll systemische Fallbesprechung Im fünften Teil unserer Serie geht es um eine fremdaggressive Patientin, die um Aufmerksamkeit ringt und keine

Krankheitseinsicht zeigt. Lesen Sie, wie das Pflegeteam es schafft, mithilfe der systemischen Fallbesprechung neue Perspektiven für den Umgang mit der Patientin zu entwickeln. Auch hier möchten wir Sie wieder einladen, sich selbst in die verschiedenen Rollen hineinzuversetzen. Fallen Ihnen weitere Lösungswege ein?

Dann schreiben Sie uns an psych.pflegeheute@thieme.de.

- Tobias Henrich -

Im Rahmen der Psychiatrischen Fachweiterbildung an der Unimedizin Mainz erlernen wir, eine systemische Fallbesprechung $(\rightarrow$ Info) zu leiten, um diese später bei Problemen zur Lösungssuche in der Praxis anwenden zu können. Nicht nur wir im Kurs profitieren durch den noch sehr ungewohnten Perspektivenwechsel. Auch der Patientin kommt es zugute, dass wir uns selber kritisch reflektieren und dadurch der Pflegeprozess nachhaltig verbessert werden kann.

\section{Falldarstellung Frau L.}

Die Falleinbringerin berichtet über die 32-jährige Patientin Frau L., die in den letzten drei Jahren bereits sechs Mal stationär aufgenommen wurde. Durch ständige, körperlich aggressive Übergriffe in der Öffentlichkeit ist sie derzeit für sechs Monate untergebracht. Seit zirka zwei Monaten ist sie nun stationär.

Frau L. ist derzeit ohne festen Wohnsitz. Ihre Wohnung wurde gekündigt. Eine betreute Wohneinrichtung wird derzeit durch den gesetzlichen Betreuer gesucht.

Pflegeproblem: Häufiges fremdaggressives Verhalten gegen Mitpatienten und/ oder Pflegepersonal.

Medizinische Diagnose: F20.0 Paranoide Schizophrenie

Problemschilderung: Frau L. zeigt im stationären Rahmen ein eingebundenes Bild mit infantilem Verhalten. Es kommt immer wieder zu Provokationen oder tät-

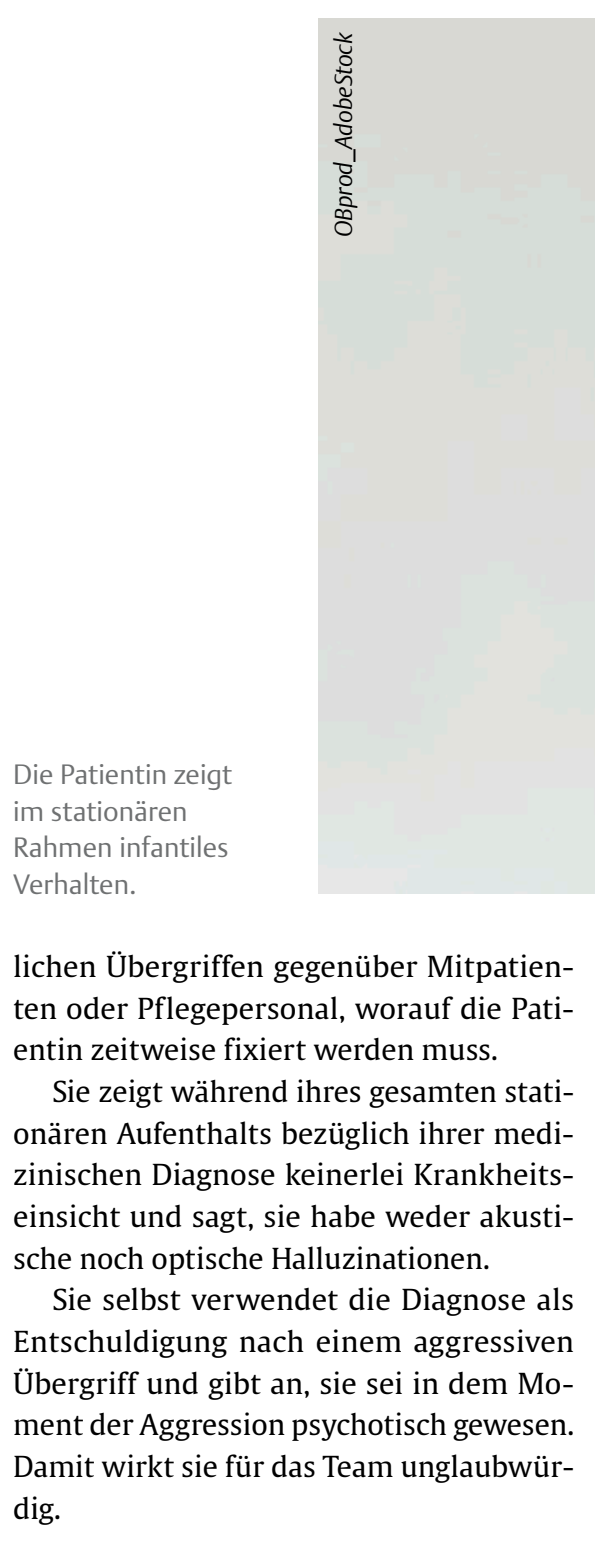

Zeitweise nehmen die Provokationen je nach Zusammensetzung der Mitpatienten zu oder $\mathrm{ab}$.

Frau L. wird derzeit lediglich mediziert, im Umgang mit ihr sieht sich das Pflegeteam ratlos.

Problemüberschrift (aus Patientensicht): Wieso werde ich ständig übergriffig?

Verlauf der systemischen Fallbesprechung

Die Falleinbringerin doppelt eine ausgewählte Person in die Rolle der Patientin ein, indem sie ihre Hände auf die Schul- 
Die systemische Fallbesprechung

Fordernde und schwierige Pflegeprobleme treten im Umgang mit Patienten immer wieder auf. Um diese zu reflektieren und zu lernen, damit umzugehen, haben Lehrende und Lernende im Weiterbildungslehrgang Psychiatrische Pflege an der Universitätsmedizin Mainz die systemische Fallbesprechung ins Leben gerufen. Damit gelingt es, die Probleme aus der Perspektive der Betroffenen zu erleben und Lösungen oder Wege zu finden, die aus der Vorstellung von Patient und Pflege bisher nicht möglich schienen.

Dabei ist es zunächst wichtig, sich in die Welt des Anderen so einzufühlen, dass wir verstehen können, was ihn so leben lässt, wie er lebt. Das ist der Ausgangspunkt, um aus einer geänderten Perspektive Lösungsmöglichkeiten zu suchen.

Der Blick bezieht die Familie, die Behandler und die Lebensumwelt ein. Durch das Eindoppeln als Technik des pädagogischen Rollenspiels (wir versetzen einen Kollegen in die Rolle unseres Patienten oder eines anderen Kollegen) werden die Gefühle durch Übertragung wach und können veräußert werden.

Der pädagogisch-pflegerische Blick öffnet sich durch die Handlungsorientierung. Aus den Blickwinkeln und dem Fachwissen sowie dem emotionalen Verständnis können wir psychiatrisch-pflegerisches Vorgehen für die Zukunft ableiten. Es verbinden sich pädagogische und die Resilienz stärkende Ideen, um Optionen für schwierige Bedingungen zu bekommen.

Der Stundenablauf wird wie folgt durchgeführt:

Informationen sammeln: Der Falleingeber berichtet über das Pflegeproblem oder die Pflegediagnose und stellt den Patienten biografisch mit Pflegeanamnese kurz vor. Er bestimmt eine Problemüberschrift.

Durchführungspraxis: Der Falleingeber stellt den Patienten aus der Sicht des

Patienten vor. Ein Kollege im Fallteam wird in den Patienten eingedoppelt, das heißt, er übernimmt die Rolle des Patienten.

Es gibt Rückmeldungen vom eingedoppelten Kollegen sowie aus dem Fallteam aus allen möglichen Perspektiven, die benannt wurden, zum Beispiel Falleingeber, Patient, Freunde, Familie, Team.

Die Gruppe macht Angebote zu möglichen Vorgehensweisen.

Der Falleingeber wählt aus den Rückmeldungen diejenigen aus, die für eine weitere Begleitung des Patienten interessant sind.

Der Falleingeber gibt im nächsten Seminar eine kurze Rückmeldung, wie die neue Intervention wirkt.

tern der Eingedoppelten legt und nun aus der Sicht von Frau L. spricht. Der Moderator hat die Möglichkeit, Fragen zu stellen, um die Person besser verstehen zu können.

\section{Rückmeldung der Gefühle von der Falleinbringerin als Frau L.}

Ich bin Frau L., 1,64 Meter groß, habe braune, lange Haare und eine normales Gewicht. Ich suche häufig den Kontakt zum Pflegepersonal, dies grenzt mich oft ein, untersagt mir vieles. Auf Station ist mir oft langweilig, ich habe manchmal nur eine Therapie am Tag. Ich fühle mich eingeschlossen und abgegrenzt.

Oft werde ich bestraft, wenn ich jemanden angegriffen habe, dabei wollte ich nur die Aufmerksamkeit des Pflegepersonals.

\section{Klärende Fragen des Moderators \\ Was wünschen Sie sich?}

Ich wünsche mir das, worauf ich gerade Lust habe. Ich kann es nicht leiden, wenn andere versuchen, mir Regeln zu geben. Ich möchte die Regeln selbst bestimmen.

Manchmal muss man sich aber doch den Gegebenheiten anpassen. Haben Sie diesbezüglich schon mal das Gespräch gesucht?

Ja, aber ich habe hier keine Alternativen außer rauchen, essen und schlafen. Die eine Therapie am Tag macht mir auch keine besonders große Freude.

Fühlen Sie sich verantwortlich für die Dinge, die Sie tun?

Ja, aber die Dinge, die ich mache, sind ja nicht schlimm. Ich mache ja auch nur was, wenn es nicht so läuft, sozusagen als Bearbeitungsstrategie.

\section{Was machen Sie denn?}

Ich stehe viel an der Tür, versuche die Gelegenheit zu nutzen, um rauszukommen. Manchmal mische ich mich auch einfach in Gespräche ein, um meine Präsenz zu zeigen.

Wenn ich dann nicht genug Aufmerksamkeit bekomme, mache ich auch schon mal was kaputt oder schlage einen Mitpatienten. Das ist die Sprache, die ich spreche, dann kommt eine Reaktion.

Sie sind also sehr interessiert daran, Aufmerksamkeit zu bekommen?

Ich denke schon. Andere empfinden es aber eher als Provokation. Ich setze es ein, um Aufmerksamkeit zu bekommen. Meist wird sich ja dann auch gekümmert.

Fühlen Sie sich oft sich selbst überlassen?

Ich hätte gern jemanden, der mich an die Hand nimmt, da ich nicht gern Verantwortung übernehme.

Fühlen Sie sich wie 32 Jahre, da Ihr Verhalten oft kindliche Züge aufweist?

Ich möchte meine eigenen Entscheidungen treffen und diese unbedacht einsetzen, egal welche Konsequenz sie haben. Andere sollen es ausräumen.

Wer hat das früher für Sie gemacht?

Früher war ich meistens bei den Großeltern, da ich viel Streit mit meinen Eltern hatte. Das Verhältnis zu meinen Eltern war immer schwierig und ist es auch heute noch. Sie haben mich nie ernst genommen und haben manchmal getrunken.

Sie sind auch jetzt meist sauer, nur weil ich anrufe, um von ihnen Geld oder Süßigkeiten zu bekommen. Das kann ich nicht nachvollziehen. Wenn sie dann zu Besuch hier waren, muss ich oft weinen. Es gab auch schon Gespräche mit den Ärzten, meinen Eltern und mir, aber außer Vorwürfen kam dabei nichts raus.

\section{Wie war die Zeit bei den Großeltern?}

Die Zeit war schön, geordnet und strukturiert.

Wie ist der Kontakt zum Pflegepersonal?

Mit den Schwestern, die etwa in meinem Alter sind, ganz gut, die sind auch nicht so streng wie die älteren. Die lassen mich auch schon mal was zu essen bestellen, wenn das Klinikessen nicht schmeckt.

Sie können also für sich sorgen?

Ja, meine Eltern bringen ja Geld. 
Sie wissen schon, wie Sie es anstellen, damit Ihre Wünsche erfüllt werden?

Ja. Ich habe auch viele Freunde, auch ehemalige Patienten. Die werden aber hier nicht reingelassen, dann werde ich böse.

Was müssten wir, Sie und Ihre Eltern tun, damit es auf Station besser läuft?

Ich bräuchte mehr Beschäftigung. $\mathrm{Zu}$ viel Zeit ist ungenutzt. Ich liege viel im Bett. Auch wenn ich Musik höre, wird es mir oft vom Personal leiser gedreht.

\section{Rückmeldung der Gefühle von der Falleinbringerin}

Ich als Frau L. fühle mich unverstanden. Alles was ich mache bringt eine meist negative Reaktion mit sich. Alles wird auf die Goldwaage gelegt und überbewertet. Es wird nicht mehr sachlich geschaut, weil alle nur noch genervt von mir sind. Die Abstände der Fixierungen werden immer kürzer. Ich weiß nicht, wo ich hingehöre, fühle mich verloren.

\section{Beobachter der zweiten Ordnung}

Nun gibt die eingedoppelte Person Rückmeldung über ihre Gefühle, welche sie empfunden hat, während die Falleinbringerin über sie berichtet hat.

Rückmeldung des Eingedoppelten als Frau L.: Es fiel mir schwer, mich in die Situation hinein zu fühlen, da die Situation sehr chaotisch auf mich wirkt. Ich möchte nicht mehr eingesperrt sein, nicht mehr in der Klinik, aber ich habe auch kein konkretes Ziel.

Grundsätzlich weiß ich, mich an Regeln zu halten, breche diese dann aber, weil ich Aufmerksamkeit suche und brauche.

\section{Rückmeldung der Gefühle aus der Gruppe}

Nun versetzt sich die beobachtende Gruppe in die Gefühle unterschiedlicher Personen, zum Beispiel der Betroffenen, der Angehörigen oder des Personals, hinein:

- Ich, als Frau L., kenne keine Strukturen, keine familiäre Bindung, keine zwischenmenschlichen Regeln.

- Ich, als Frau L., fühle mich mit übergestülpten Regeln überfordert.

- Wir, als Eltern, versuchen, mit unserem Geld etwas gutzumachen.

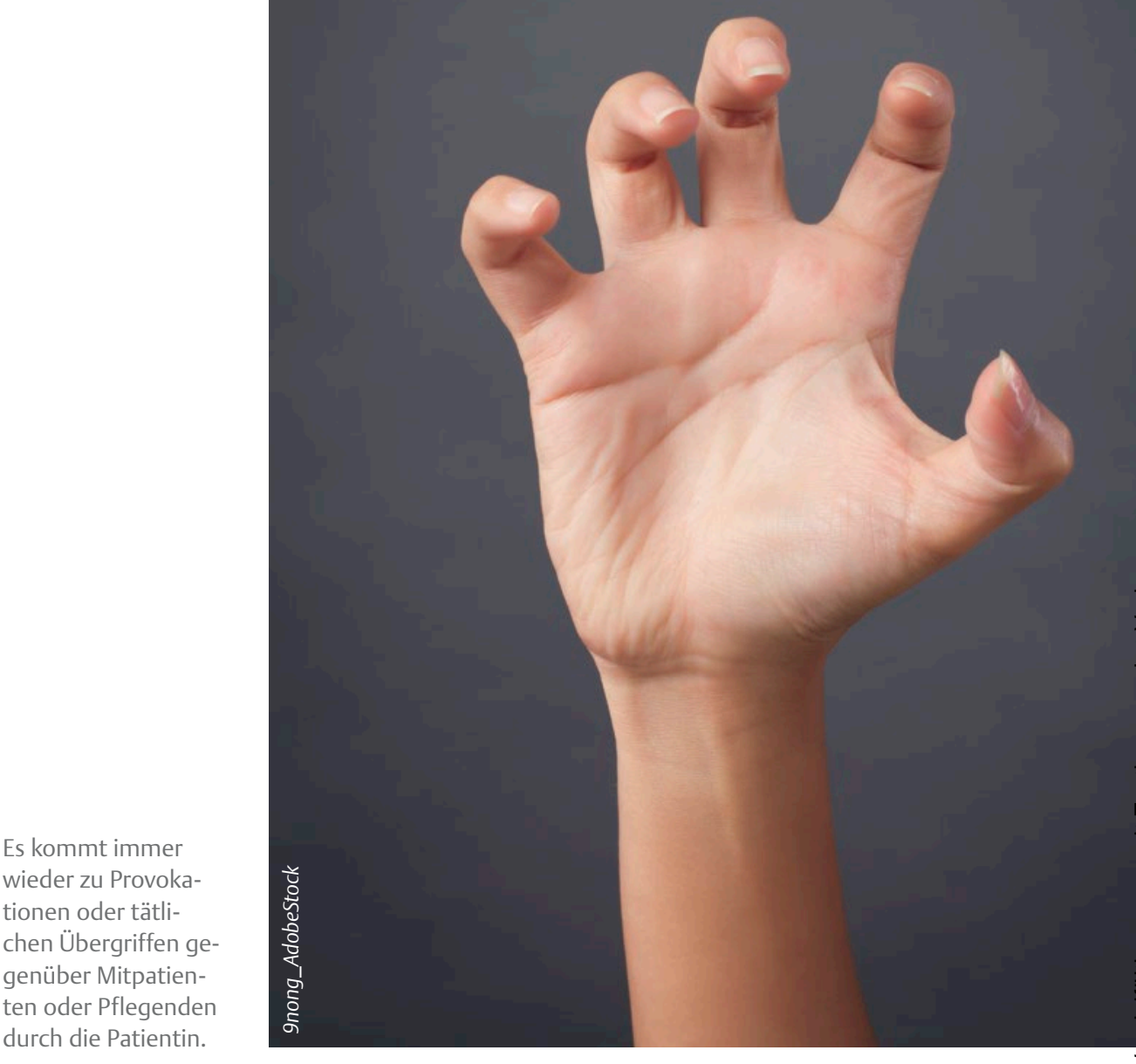

- Wir, als Eltern, sind froh, dass wir die Verantwortung abgeben konnten, weil wir überfordert sind.

- Wir, als Eltern, wollten das Kind nicht.

- Ich, als Frau L., finde gleichaltriges Personal ganz nett.

- Ich, als Frau L., möchte die Dinge gerne so, wie ich sie haben will.

- Ich, als Frau L., möchte gerne Aufmerksamkeit.

\section{Angebote von Optionen}

Rückmeldungen und Lösungen aus der Gruppe:

- Angebot von psychologischen Gesprächen, um eine Psychotherapie zu ermöglichen, in der die Kindheit aufgearbeitet wird.

- Verhalten der Patientin an ein Belohnungssystem knüpfen. Bei positivem Verhalten Privilegien einräumen, die der Patientin Freude und Entlastung bereiten.

- Patientin immer wieder positiv verstärken um das Erwachsenen-Ich positiv darzustellen.

- Bezugspflege, um der Patientin eine feste Bezugsperson zu bieten.

- Abstimmen der Therapieangebote, um den Bedürfnissen der Patientin gerecht zu werden.

\section{Mögliche Neukonstruktion}

Die Falleinbringerin bedankt sich für die Lösungsvorschläge. Sie bevorzugt den Lösungsvorschlag, das Verhalten der Patientin an ein Belohnungssystem zu knüpfen. Des Weiteren möchte sie in Absprache mit dem therapeutischen Team und der Patientin das Therapieangebot auf die Bedürfnisse von Frau L. abstimmen.

Die Namen und Orte wurden von der Redaktion geändert.

Dieses Protokoll ist entstanden im Weiterbildungslehrgang Psychiatrische Pflege der Universitätsmedizin Mainz.

\section{Autor}

Tobias Henrich

Krankenpfleger, Fachkrankenpfleger für Rehabilitation, Praxisbegleiter für Basale Stimulation in der Pflege, Pflegeexperte für Patientenedukation, Praxisanleiter für Gesundheitsberufe, aktuell in der Weiterbildung für Psychiatrische Pflege

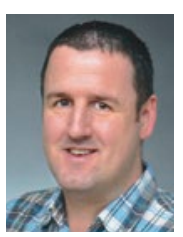

\section{Bibliografie}

DOI 10.1055/s-0043-124843

PPH 2018; 24: 63-65

(c) Georg Thieme Verlag KG

Stuttgart · New York · ISSN 0949-1619 E-Mail: tobias.henrich78@web.de 